\title{
An Improved Image Re-indexing Technique by Self Organizing Motor Maps
}

\author{
Sebastiano Battiato, Francesco Rundo, and Filippo Stanco \\ Dipartimento di Matematica e Informatica, \\ University of Catania, Viale A. Doria, 6, 95125 Catania, Italy \\ \{battiato, rundo, fstanco\}@dmi.unict.it \\ http://iplab.dmi.unict.it
}

\begin{abstract}
The paper presents a novel Motor Map neural network for re-indexing color mapped images. The overall learning process is able to smooth the local spatial redundancy of the indexes of the input image. Differently than before, the proposed optimization process is specifically devoted to re-organize the matrix of differences of the indexes computed according to some predefined patterns. Experimental results show that the proposed approach achieves good performances both in terms of compression ratio and zero order entropy of local differences. Also its computational complexity is competitive with previous works in the field.
\end{abstract}

\section{Introduction}

Color-mapped images [1] make use of an index map to store the different involved colors maintaining for each pixel the location of the corresponding index. Reindexing techniques reduce the local indexes redundancy try to find the optimal reordering avoiding to consider all possible color indexing ( $M$ ! for an image with $M$ colors). The existing re-indexing algorithms are devoted to obtain color and index similarity [2. The color based solutions [3, 4, 5, 6, 7, 8, assign consecutive symbols to visually similar colors according to some heuristic measures (typically, the indexes are sorted by luminance order [3]). Alternatively, index based methods [9, 10, 11, 12] are guided by both information theory and local adaptive considerations even if they have an intrinsic inefficiency to numerically optimize the palette re-indexing. To overcome this problem different heuristics have been proposed [2,13,14].

Recently, in [15, 16] improvements have been obtained by pre-processing the indexed input image with respect to the available encoder. Such methods provide only a much more sophisticated "coded" representation and they cannot be classified as methods for re-indexing.

In [17, a Motor Map (MM) neural network [18] has been used to properly learn the shape palette clustering for searching (in the output stage of the network) the optimum indexing scheme. The overall results have proved the ability of the self organizing process to obtain effective results.

In this paper we propose a method that outperform existing approaches by properly design a MM neural network that works and manipulates directly the

A. Trémeau, R. Schettini, and S. Tominaga (Eds.): CCIW 2009, LNCS 5646, pp. 62 -70, 2009.

(C) Springer-Verlag Berlin Heidelberg 2009 
matrix of differences of the input image. This allows to exploit the solution space in a profitable way. As in [17] the ability of MM to find an optimal solution without requiring the knowledge of the underlying model has been crucial in this context. The overall performances have been evaluated by considering the same repository used in [2] that contains images with different size and number of colors. Experimental results show how the proposed method outperforms previous results in the field both in terms of overall compression ratio and residual zero-order entropy of the images with the best performance even in terms of computational complexity.

The paper is structured as follows. Section 2 introduces the re-indexing problem, while the proposed approache is detailed in Section 3 . Experimental results are presented in Section 4. Conclusions are drawn in Section 5

\section{Problem Formulation}

The re-indexing problem can be stated as expressed in [2,14]. Let $I$ be an image of $m \times n$ pixels, and $M$ be the number of distinct colors. $I$ can be represented as $I(x, y)=P\left(I^{\prime}(x, y)\right)$, where $P=\left\{S_{1}, S_{2}, \ldots, S_{M}\right\}$ is the set of all the colors in $I$, and $I^{\prime}$ is a $m \times n$ matrix of indexes in $\{1,2, \ldots, M\}$. An image represented in such a fashion is called indexed image (or color mapped image) and $P$ is its palette. For indexed image, an ordered scan of the indexes in $I^{\prime}$ named $p_{1}, \ldots$, $p_{m \times n}$ is usually performed. The residual entropy of local differences can be considered to estimate the overall "energy" of the signal. The information needed to reconstruct the original image is: 1$)$ the color of pixel $p_{1} ; 2$ ) a table providing the correspondence between colors $S_{1}, S_{2}, \ldots, S_{M}$ with index $i_{1}, i_{2}, \ldots, i_{M} ; 3$ ) the set of differences: $D\left(I^{\prime}\right)=\left\{d_{x, y} \mid x=1,2, \ldots, m y=1,2, \ldots, n\right\}$ where each $d_{x, y}$ is a local difference obtained by considering some specific patterns as better specified below.

Information theory states that any lossless scheme to encode the set of differences $D\left(I^{\prime}\right)$ requires a number of bits per pixel (bpp) greater or equal to the true source entropy. For our purposes it is sufficient to measure the zero-order entropy of the statistical distribution of $D\left(I^{\prime}\right)$ properly managing the proposed optimization process. If indexes $i_{1}, i_{2}, \ldots, i_{M}$ are ordered so as to produce an almost uniform distribution of values $d_{x, y}$ the entropy value will be large. Conversely, a zero-peaked distribution in $D\left(I^{\prime}\right)$ gives a lower entropy value. Hence, finding an optimal indexing scheme is a crucial step for any lossless compression of indexed images.

\section{The MM Re-indexing Algorithm}

\subsection{MM for Re-indexing}

The re-indexing problem is addressed in literature by means of two main approaches [2]: the ones based on color information and the other ones based on the information retrieved from index matrix . In [17] we have showed that MM 
is able to reach high performance in searching optimum indexing scheme, by means of color based training set. In [17] we show how MM is also able to reach near "optimum" indexing scheme by means of index based training set. This shows the high capability of MM to solve complex problem such as the image reindexing. The self organization capability is strongly related to the "knowledge" of problem to be solved. More information and detailed problem descriptions increase the ability of the MM to provide right solution for the problem. The results shows that the optimum re-indexing scheme is "more" related to index based information with respect to the color palette as the obtained results outperform the same obtained with previous version [17. This is a result which has not been discovered till now.

The proposed algorithm is based on the ability of the MM neural network to learn the "features" of the input pattern providing an appropriate output stimulus. The learning process has been modified respect to the algorithm in [17] in order to adapt itself to reduce the local spatial redundancy. We assume that the MM can learn the main information about the entropy minimization of the source image $I$ if it is trained by the elements of the matrix $D$ and not by using only the color of the index. In this way the structural characteristics (e.g., presence of edges, flat areas, etc. ) of the underlying image are properly addressed. As shown in the experimental section, the final matrix of indexes obtained at the end of the optimization process is clearly smoother than before allowing to achieve better compression results. The overall approach can be described by the following steps.

Step 1. The topology of the MM has been established by making use of a lattice structure of $(m-1) \times(n-1)$ neurons, where $m$ and $n$ are the original dimensions of the input image $I$. Let $Q=(m-1) \times(n-1)$ be the number of neurons of the MM. Each neuron is composed by an input weight $w_{i}^{i n}, i=1, \ldots, Q$ with $w_{i}^{i n} \in[0,1]$ and output weight $w_{i}^{\text {out }}, i=1, \ldots, Q$ and a variable $b_{i}, i=1, \ldots, Q$ which stores the average increasing of the reward function. The range of the $w_{i}^{\text {out }}$ values is $[1, \ldots, M]$. In our case, the following reward function has been chosen:

$$
\text { Reward }=-\left(\sum D\left(I^{\prime}\right)\right)^{2}
$$

The above reward is strictly proportional to the zero-order entropy of the differences matrix $D\left(I^{\prime}\right)$. Moreover, the selection of the above reward function leads the MM to find an optimum palette index scheme which minimize the entropy of the image and the relative compression ratio. Regarding the output stimulus produced by the MM during the learning phase, in this work, it has been forced equal to $w_{i}^{\text {out }}$. The $w_{i}^{\text {out }}$ will be equal to a random index generated during the learning process when the corresponding neuron wins. Before to start the learning phase, the MM (both input layer and output layer) is initialized randomly. Before starting the learning phase, a palette pre-processing is applied (i.e., the corresponding lightness factor of the input colors are sorted in increasing order).

Step 2. Let $D\left(I^{\prime}\right)$ the matrix of differences computed starting form the index matrix $I^{\prime}$. The set of differences $D\left(I^{\prime}\right)$ has been computed by using different local pattern configurations [19]: 


$$
\begin{gathered}
D_{V_{1}}\left(I^{\prime}\right)=\left\{I_{x, y}^{\prime}-I_{x, y-1}^{\prime}\right\} \\
D_{V_{2}}\left(I^{\prime}\right)=\left\{I_{x, y}^{\prime}-I_{x-1, y}^{\prime}\right\} \\
D_{V_{3}}\left(I^{\prime}\right)=\left\{\frac{2 * I_{x, y}^{\prime}-I_{x, y-1}^{\prime}-I_{x-1, y}^{\prime}}{2}\right\} \\
D_{V_{4}}\left(I^{\prime}\right)=\left\{\frac{3 * I_{x, y}^{\prime}-I_{x, y-1}^{\prime}-I_{x-1, y}^{\prime}-I_{x-1, y-1}^{\prime}}{3}\right\}
\end{gathered}
$$

where $x, y$ are the corresponding valid indexes in the original $m \times n$ image $I$. Just using an initial configuration $V_{1}$ and considering for simplicity only the absolute value of differences we obtain the initial configuration.

Step 3. Each element of the matrix $D$, normalized in the range $[0,1]$, is fed to the input layer of the MM, one element at each iteration, searching the winner neuron (i.e., the neuron which has the minimum value of the following value):

$$
v_{i}=\left|d_{x, y}-w_{i}^{i n}\right| \quad i=1,2, \ldots, Q
$$

where $x=1,2, \ldots, m$; and $y=1,2, \ldots, n$.

The winner neuron provides an updating of the output stimulus which is, in this case, a new index (i.e., a new index for the related color on the corresponding palette performing also the related swaps on the indexes image). The new index $i n d_{x, y}^{r a n d}$ is generated randomly in the range $[1,2, \ldots, M]$. After the index updating, the new reward function can be computed updating only the elements in the associated matrix $D\left(I^{\prime}\right)$ which have been involved in the indexes swapping, in order to speed up the algorithm execution time. The $\Delta$ Reward will be computed as:

$$
\Delta \text { Reward }=\left(\text { Reward } d^{\text {new }}-\text { Reward }^{\text {old }}\right)^{2}
$$

The average increasing of the reward function is weighted by the $b_{w i n}^{\text {new }}$ :

$$
b_{\text {win }}^{\text {new }}=b_{\text {win }}^{\text {old }}+\rho\left(\Delta \text { Reward }-b_{\text {win }}^{\text {old }}\right)
$$

where $\rho$ is a positive value related to the smoothing action.

Step 4. If the $\Delta$ Reward $\geq b_{w i n}^{\text {new }}$ and the new current entropy (really the new current sum of absolute differences) is better than the already ones processed till now, the new index scheme will be accepted and the weights of the winner neurons will be updated:

$$
\begin{aligned}
& w_{\text {win }}^{\text {in }}(t+1)=w_{w i n}^{\text {in }}(t)+\eta\left(d_{x, y}-w_{\text {win }}^{\text {in }}(t)\right) \\
& w_{\text {win }}^{\text {out }}(t+1)=i n d_{x, y}^{\text {rand }}
\end{aligned}
$$

where $\eta$ is the learning rate factor. After that, the learning steps (from 2 to 4 ) is repeated until the stop criteria is verified. Conversely, the new index scheme will 


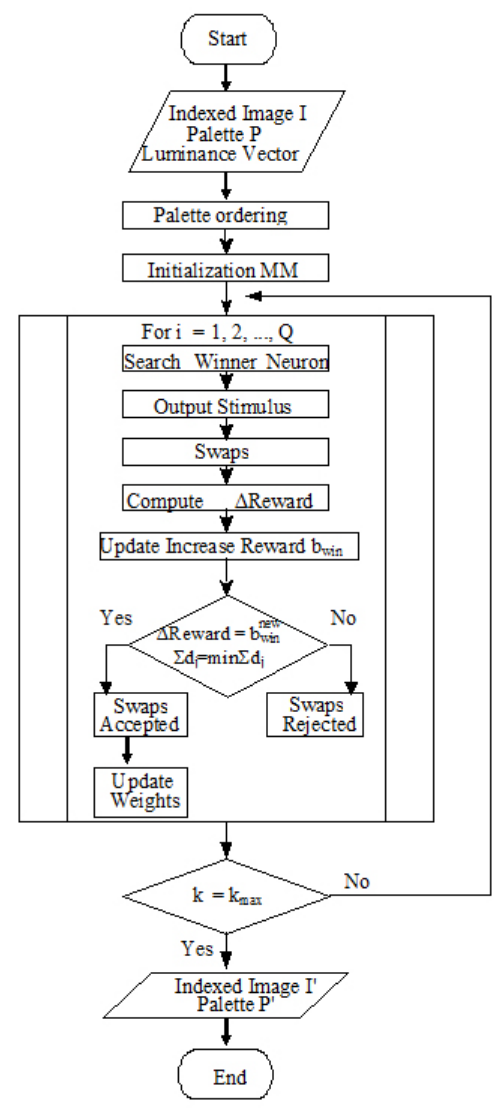

Fig. 1. Flow chart of the proposed MM algorithm

be rejected and the previous ones will be restored. Fig. 1 reports a schematic representation of the overall process. In the proposed MM architecture, the neuron has not an adaptive neighboring and the learning rate remains constant during all the learning phase. The stop of the learning process is reached when the computed entropy is less or equal to a specific lower bound value or after a fixed number of epochs (an epoch is a number of cycles needed for presenting all the input patterns to neural network). To avoid local minimum a standard random scrolling of the weights are employed; it provides a perturbation of the neurons by using a gaussian random variable.

\subsection{Algorithm Parameters}

The MM parameters are chosen according to trial-and-error policies as well as heuristic considerations. In particular, we have chosen $\eta=0.95$ and $\rho=0.90$. Maximum number of learning cycle $k$ has been heuristically set to $10^{6}$ in all involved experiments. 


\subsection{Computational Complexity}

Let $M$ the overall number of involved colors of an input image $I$ having $N=m \times$ $n$ pixels. The proposed technique requires a preprocessing phase devoted to sort the input colors according to their lightness factor. Each learning cycle have to compute the reward function, by considering just a single index swap in the matrix index $D\left(I^{\prime}\right)$. The overall computational complexity is $O(M \log M)+O\left(k N^{2}\right)$ where $k$ is the number of learning cycles. Further experiments will be devoted to measure the average number of iteration $k$ required to achieve convergence. In the current implementation we have used $10^{6}$ as maximum possible value. As showed in [17] the proposed MM re-indexing is competitive with the state of the art solutions: $O(M \log M)$ for luminance order, $O\left(M^{2} \log M\right)$ for Battiato's approach [14, $O\left(M^{3}\right)$ for Zeng's [13] and its modification [19], and $O\left(M^{4}\right)$ for Memon [12. See [2,17,20] about interesting considerations over computational complexity for re-indexing process.

\section{Experimental Results}

In order to check the performances of the $\mathrm{MM}$ as palette re-indexing algorithm (called MMap_new), we propose the comparison between our method and the most important reordering methods [12,13, 14, 17, 19. For sake of comparison, the dataset used is the same of 2. In our experiments we have used the following two groups: a set of natural images also known as 'kodak' database, and a set of popular natural images. These contain quantized version (non-dithered) of the same images with 64 colors. Table 1 reports the final residual entropy of the local differences by making use of pattern $V_{i}$ with $i=1, \ldots, 4$ applied to the two datasets considering the current approach MMap_new and MMap [17. The proposed new approach clearly outperform [17] in all cases. As stated above, the MMap_new re-indexing scheme has been applied by computing the residual zero order entropy of local differences $D\left(I^{\prime}\right)$ by considering first the pattern $V_{1}$ and then re-applying the overall process just considering pattern $V_{2}$. A useful comparisons of the final entropy values between the proposed method MMap_new and the others is reported in Table 2 .

Table 1. Residual entropy of local differences computed by using $V_{i}, i=1, \ldots, 4$

\begin{tabular}{|l|r|r|r|r|}
\hline & \multicolumn{4}{|c|}{ MMap [17 } \\
\hline & V1 & V2 & V3 & V4 \\
\hline Natural1 & $\mathbf{2 , 5 3 4}$ & 2,693 & 2,621 & 2,735 \\
Natural2 & $\mathbf{2 , 7 5 3}$ & 2,787 & 2,774 & 2,874 \\
\hline \hline & \multicolumn{4}{|c|}{ MMap_new } \\
\hline & V1 & V2 & V3 & V4 \\
\hline Natural1 & $\mathbf{1 , 4 1 6}$ & 1,512 & 1,496 & 1,545 \\
Natural2 & 2,739 & $\mathbf{2 , 7 3 7}$ & 2,741 & 2,834 \\
\hline
\end{tabular}




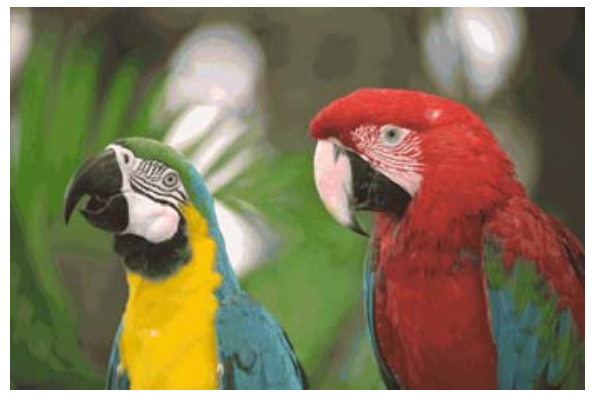

(a)

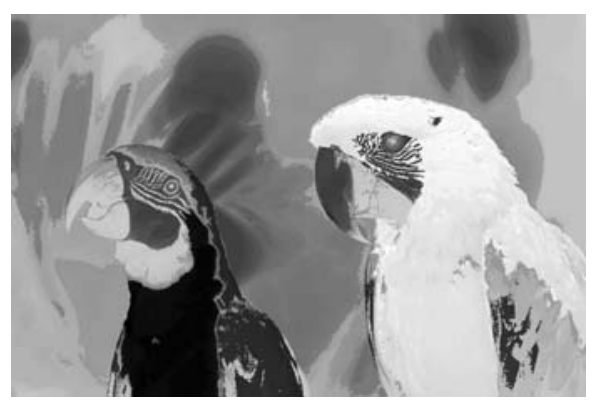

(c) Entropy $=1,556$

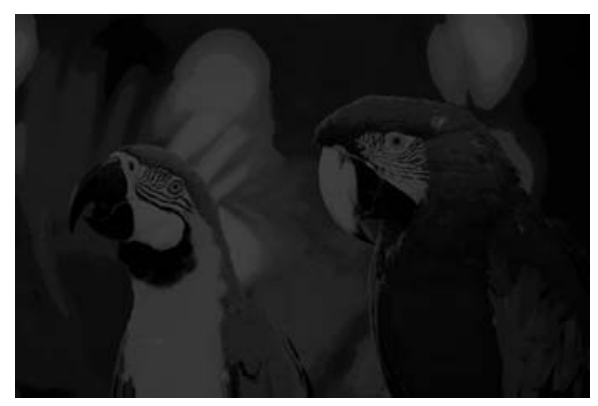

(e) Entropy $=1,676$

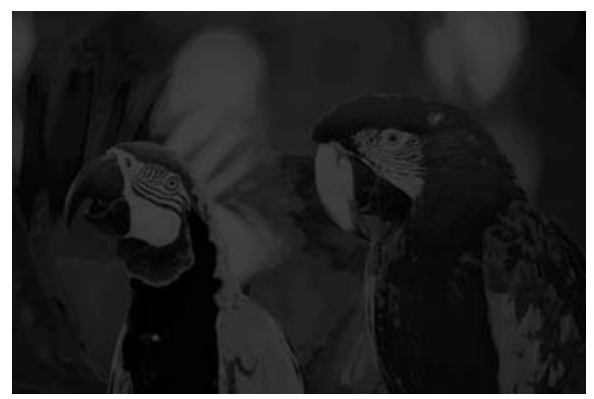

(b) Entropy=1,785

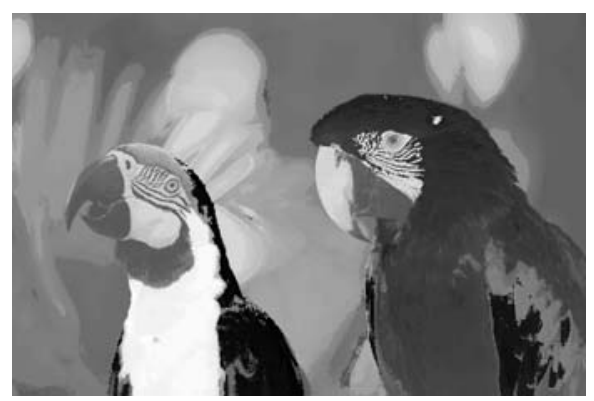

(d) Entropy=1,523

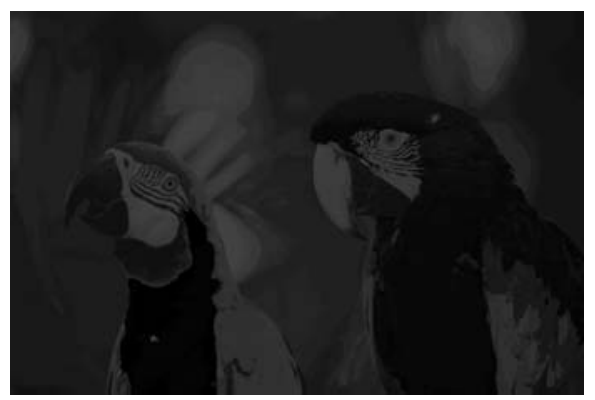

(f) Entropy $=0,889$

Fig. 2. Different re-indexing schemes. (a) Color image; (b) Original indexes; (c) Memon 12]; (d) mZeng [19; (e) MMap [17]; (f) MMap_new.

Finally, Table 3 reports the bits rate in terms of bpp (bit per pixels) obtained by lossless compression of the datasets after palette reordering with JPEG-LS1, and PNG2, respectively. By using all coding engines, the values of bpps of the proposed approach are considerable lower than the other methods, in some cases the differences are substantial. Finally, we show the final re-ordered palette for a

\footnotetext{
${ }^{1}$ SPMG/JPEG-LS Encoder 1.0.

2 Matlab 7.0.1.
} 
Table 2. Residual zero-order entropy of images before and after using the palette reordering methods (Memon [12, Zeng [13, mZeng [19, Battiato [14] and MMap [17])

\begin{tabular}{|l|r|r|r|r|r|r|r|r|}
\hline & Random & Lum. & Memon & Zeng & mZeng & Battiato & MMap & MMap_new \\
\hline Natural1 & 3,616 & 3,101 & 2,444 & 2,491 & 2,491 & 2,576 & 2,534 & $\mathbf{1 , 4 1 6}$ \\
\hline Natural2 & 4,414 & 3,944 & 3,100 & 3,236 & 3,226 & 3,370 & 3,133 & $\mathbf{2 , 7 3 9}$ \\
\hline
\end{tabular}

Table 3. Lossless compression results in bit per pixel, obtained with Jpeg-LS and PNG applied to the indexed images after using the palette re-ordering methods (Memon 12, Zeng [13], mZeng [19, Battiato 14] and MMap [17])

\begin{tabular}{|l||r|r|r|r|r|r|r|r|r|}
\hline & Type & Random & Lum. & Memon & Zeng & mZeng & Battiato & MMap & MMap_new \\
\hline \multirow{2}{*}{ JPEG-LS } & Natural1 & 3,661 & 3,002 & 2,641 & 2,804 & 2,709 & 3,144 & 2,710 & $\mathbf{2 , 4 3 1}$ \\
& Natural2 & 3,967 & 3,069 & $\mathbf{2 , 7 9 3}$ & 3,060 & 2,912 & 3,281 & 2,921 & 2,998 \\
\hline PNG & Natural1 & 4,234 & 3,613 & 3,438 & 3,497 & 3,529 & 3,116 & 3,009 & $\mathbf{2 , 0 9 1}$ \\
& Natural2 & 5,396 & 4,715 & 4,558 & 4,545 & 4,671 & 4,130 & $\mathbf{3 , 3 0 7}$ & 3,424 \\
\hline
\end{tabular}

single image just to visually evaluate the smoothness obtained with the different methods (Fig. 2).

\section{Conclusion and Future Work}

Palette reordering is a very effective approach for improving the compression of color-indexed images. In this paper, we described a technique that shows a good performance on the optimum palette scheme generation making use of a self organizing MM that works directly on the spatial smoothness of the matrix of differences of the input image. Preliminary experiments confirm the real effectiveness of the proposed approach. Future works will be devoted to extend the experimental phase to larger dataset. Also the possibility to design proper reward function, specifically addressed to the specific codec engine will be considered.

\section{References}

1. Battiato, S., Lukac, R.: Color-Mapped Imaging. In: Furth, B. (ed.) Encyclopedia of Multimedia, pp. 83-88. Springer, Heidelberg (2008)

2. Pinho, A.J., Neves, A.J.R.: A survey on palette reordering methods for improving the compression of color-indexed images. IEEE Transactions on Image Processing 13(11) (November 2004)

3. Zaccarin, A., Liu, B.: A novel approach for coding color quantized images. IEEE Transactions on Image Processing 2, 442-453 (1993)

4. Spira, A., Malah, D.: Improved lossless compression of color-mapped images by an approximate solution of the traveling salesman problem. In: IEEE Int. Conf. Acoustics, Speech, Signal Processing, May 2001, vol. III, pp. 1797-1800 (2001)

5. Po, L.M., Tan, W.T.: Block address predictive color quantization image compression. Electron. Lett. 30(2), 120-121 (1994) 
6. Hadenfeldt, A.C., Sayood, K.: Compression of color-mapped images. IEEE Trans. Geosci. Remote Sens. 32, 534-541 (1994)

7. Lai, J.Z.C., Liaw, Y.-C.: A novel approach of reordering color palette for indexed image compression. IEEE Signal Processing Letters 14(2), 117-120 (2007)

8. Chuang, W.-H., Pei, S.-C.: A low-complexity palette re-indexing technique based on sampling-swapping. In: Proc. of IEEE International Conference on Image Processing, pp. 1029-1032 (2008)

9. Fojtik, J., Vaclav, H.: Invisible modification of the palette colour image enhancing lossless compression. In: Amin, A., Pudil, P., Dori, D. (eds.) SPR 1998 and SSPR 1998. LNCS, vol. 1451, pp. 1029-1036. Springer, Heidelberg (1998)

10. Waldemar, P., Ramstad, T.A.: Subband coding of color images with limited palette size. In: IEEE Int. Conf. on Acoustics, Speech, and Signal Processing, ICASSP 1994, April 1994, No. V, pp. 353-356 (1994)

11. Gormish, M.J.: Compression of palletized images by colour. In: Proc. of IEEE International Conference on Image Processing (1995)

12. Memon, N., Venkateswaran, A.: On ordering colour maps for lossless predictive coding. IEEE Trans. Image Proc. 5(11), 1522-1527 (1996)

13. Zeng, W., Li, J., Lei, S.: An efficient colour re-indexing scheme for palette-based compression. In: Proc. of 7th IEEE International Conference on Image Processing, pp. 476-479 (2000)

14. Battiato, S., Gallo, G., Impoco, G., Stanco, F.: An efficient re-indexing algorithm for color-mapped images. IEEE Transactions on Image Processing 13(11), 1419 $1423(2004)$

15. You, K.-S., Han, D.-S., Jang, E.S., Jang, S.-Y., Lee, S.-K., Kwak, H.-S.: Ranked image generation for arithmetic coding in indexed color image. In: Proceedings of 7th International Workshop on Enterprise networking and Computing in Healthcare Industry, HEALTHCOM, June 2005, pp. 299-302 (2005)

16. Neves, A.J.R., Pinho, A.J.: A bit-plane approach for lossless compression of colorquantized images. In: Proc. of the IEEE Int. Conf. on Acoustics, Speech, and Signal Processing, ICASSP 2006, May 2006, vol. 13 (2006)

17. Battiato, S., Rundo, F., Stanco, F.: Self organizing motor maps for color-mapped image re-indexing. IEEE Transactions on Image Processing 16(12), 2905-2915 (2007)

18. Arena, P., Fortuna, L., Frasca, M., Sicurella, G.: An adaptive, self-organizing dynamical system for hierarchical control of bio-inspired locomotion. IEEE Transactions on Systems, Man, and Cybernetics, Part B 34, 1823-1837 (2004)

19. Pinho, A.J., Neves, A.J.R.: On the relation between memon's and the modified zeng's palette reordering methods. Image Vision Comput. 24(5), 534-540 (2006)

20. Pei, S.-C., Chuang, Y.-T., Chuang, W.-H.: Effective palette indexing for image compression using self-organization of kohonen feature map. IEEE Transaction on Image Processing 15(9), 2493-2498 (2006) 